\title{
Application of speckle decorrelation method for small translation measurements
}

\author{
Pavel HORVÁth, Miroslav HRABOVSKÝ, Petr ŠMíD \\ Joint Laboratory of Optics of Palacky University and Institute of Physics of Academy of Sciences \\ of the Czech Republic, Tr. 17. listopadu 50, 77207 Olomouc, Czech Republic, \\ e-mails: horvath@optnw.upol.cz,hrabovsky@optnw.upol.cz, smid@optnw.upol.cz
}

\begin{abstract}
This paper analyses the usage of the speckle pattern decorrelation method for determination of small static and dynamic object translations. At first the philosophy of the method is presented briefly. Then relationships between the cross-correlation function and the small deformation tensor for the case of optically free space and image field are mentioned. Next, different experimental arrangements for the measurement of in-plane and normal object translations are analysed. Possible measurement ranges and sensitivities for each arrangement are discussed, too. Finally, some results of our experiments are shown.
\end{abstract}

Keywords: speckle, decorrelation, in-plane and normal translation.

\section{Introduction}

An object translation measurement is one of the often measured deformation tensor components. There are many methods that enable us to obtain the desired information. However, most of them are based on the direct contact of the measuring tool with the measured object, which can be destructive. Therefore, it is required that new ways of noncontact measurement be searched for. Optics offers suitable solutions since optical radiation can be used as a means of nondestructive measurements. One of the possibilities is a method utilizing a speckle pattern effect that occurs, for example, in holographic area as a parasitical element.

The speckle pattern [1]-[3] arises if an object with a rough surface is illuminated by a coherent optical beam. This pattern consisting of dark and bright speckles results from the interference of multiple coherent spherical waves generated by point sources forming the object surface. Since the point sources emit waves into a wide range of angles, the arising speckle field is not localized in space (e.g., one plane) but it fills the complete volume occupied by the interfering waves. A coherent light propagating through a medium with a random refractive index fluctuation or striking upon a diffuser can also form the speckle pattern. 
The method proposed for the small translation measurement is based on the fact that the speckle pattern appearing in the observation plane moves and changes its structure in consequence of object translation, rotation or deformation. The information about the state of the object surface can be obtained from the detected speckle displacement regressively. This method was first theoretically described and put into practice by Yamaguchi [4]-[7]. HRABOvskÝ et al. [8]-[13] verified and extended it later.

There are other methods for measuring small object surface changes that use interference fringes created in the speckle pattern [1], [14]. However, in some cases it is hard to localize the fringes. The present method does not use any fringes and the information about the object state is obtained statistically by means of cross-correlation function of two intensity sets recorded before and after the object deformation.

\section{Basic equations}

In order to find out the relationship between the speckle pattern displacement and the small deformation tensor components that describe the deformation state of the elementary object surface area let us adopt a geometrical arrangement according to Fig. 1 a. The rough object surface placed in the $(x, y)$ plane is illuminated with a spherical wave originating from the point source $S$ (the case of perfectly polarized and coherent light is considered). The reflected light that forms a speckle field is captured by a linear detector located in the observation (detection) plane $(X, Y)$. Furthermore, it is assumed that the real and imaginary parts of the complex amplitude of light at any point of the observation plane have Gaussian distribution with a zero mean value and intensity of the speckle spot obeys the exponential distribution. Considering the light propagation after the reflection is described with the Fresnel approximation and the rotation and the deformation components of the small deformation tensor are equalled to zero (the object is moved as a rigid body), the following relations can be derived [4], [8], [9]:

$$
\begin{aligned}
& A_{X}=a_{x}\left(\frac{L_{o} \cos ^{2} \theta_{s}}{L_{s} \cos \theta_{o}}+\cos \theta_{o}\right)-a_{z}\left(\frac{L_{o} \cos \theta_{s} \sin \theta_{s}}{L_{s} \cos \theta_{o}}+\sin \theta_{o}\right), \\
& A_{Y}=a_{y}\left(\frac{L_{o}}{L_{s}}+1\right) .
\end{aligned}
$$

Both equations are in accordance with Fig. 1a and valid for the case of optically free space since there is no optical system between the object plane and the detection plane. The quantities $A_{X}$ and $A_{Y}$ are the $x$-and $y$-components of the speckle pattern displacement vector in the $(X, Y)$ plane. These components can be determined from 


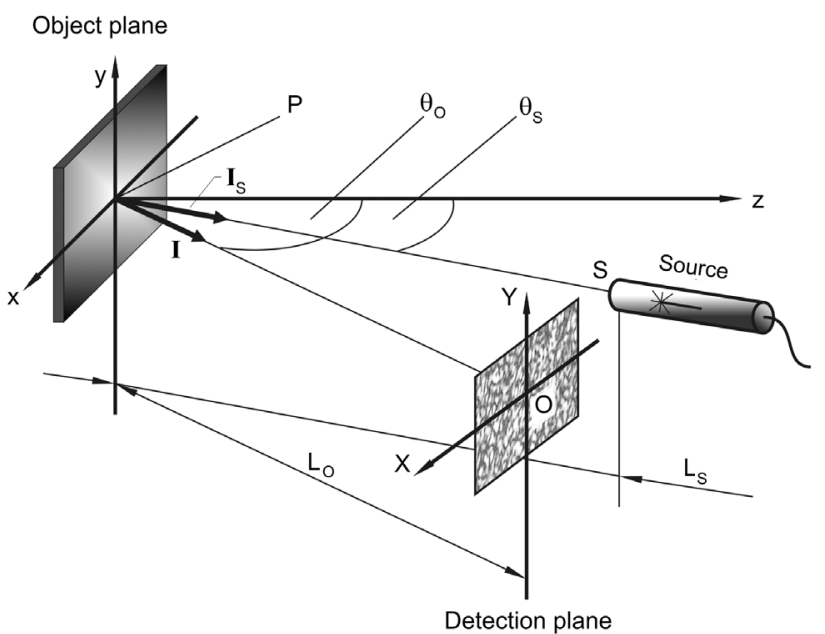

a

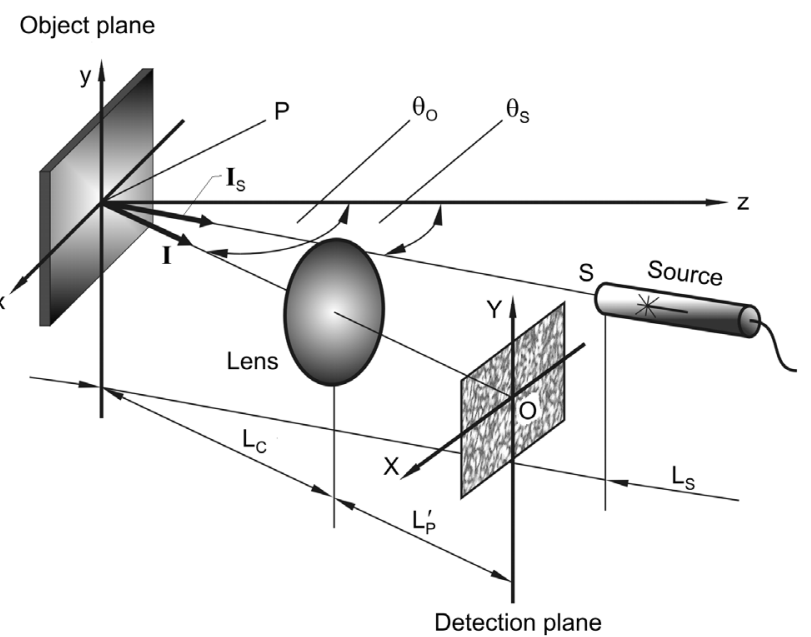

Fig. 1. Coordinate system for observation of the speckle pattern. Points $O, P$ and $S$ are located in the $(x, z)$ plane: $\mathbf{a}$ - free space, $\mathbf{b}$ - image field.

the position of the cross-correlation function maximum of fluctuations of intensity sets (represented by intensity values obtained from each pixel of the linear CCD detector) recorded in the detection plane before and after the object deformation. It is important to note that the decline of this maximum corresponds to the magnitude of change of the speckle pattern (so-called speckle decorrelation). Next, $a_{x}, a_{y}, a_{z}$ are the translation components of the small deformation tensor in the $x$ -,$y$-, $z$-axes direction. Finally, $L_{o}$, $L_{s}, \theta_{o}, \theta_{s}$ are the geometrical parameters of the optical arrangement, where $L_{o}, L_{s}$ are 
the distances between the object surface plane and the observation plane, the source respectively, $\theta_{s}$ is the angle of the illumination direction and $\theta_{o}$ is the angle of the observation direction.

If an optical system, for example, a thin lens with focal length $f^{\prime}$ (see Fig. 1b), is placed between the object plane and the detection plane (the case of image field) and ideal imaging is assumed, then Eqs. (1) and (2) can be transcribed into the following forms [4], [10]:

$$
\begin{aligned}
-\frac{A_{X} f^{\prime}}{L_{p}^{\prime}-f^{\prime}}= & -a_{x}\left(\frac{L_{c}\left(L_{p}^{\prime}-f^{\prime}\right)-f^{\prime} L_{p}^{\prime}}{L_{s}\left(L_{p}^{\prime}-f^{\prime}\right)} \frac{\cos ^{2} \theta_{s}}{\cos \theta_{o}}+\cos \theta_{o}\right) \\
& +a_{z}\left(\frac{L_{c}\left(L_{p}^{\prime}-f^{\prime}\right)-f^{\prime} L_{p}^{\prime}}{L_{s}\left(L_{p}^{\prime}-f^{\prime}\right)} \frac{\cos \theta_{s} \sin \theta_{s}}{\cos \theta_{o}}\right)+a_{z} \sin \theta_{o} \\
-\frac{A_{Y} f^{\prime}}{L_{p}^{\prime}-f^{\prime}}= & -a_{y}\left(\frac{L_{c}\left(L_{p}^{\prime}-f^{\prime}\right)-f^{\prime} L_{p}^{\prime}}{L_{s}\left(L_{p}^{\prime}-f^{\prime}\right)}+1\right)
\end{aligned}
$$

where $L_{c}$ is the distance from the thin lens to the object plane and $L_{p}^{\prime}$ is the distance from the thin lens to the detection plane. So, Eqs. (1)-(4) enable us to determine $a_{x}$, $a_{y}, a_{z}$ components of the small deformation tensor by means of the detected speckle pattern displacement both in the case of the free space and the image field. It is evident that two types of the object translation can be measured. In the case of in-plane translation (components $a_{x}, a_{y}$ are measured) the object surface is translated only in the $(x, y)$ plane. In the case of normal translation (component $a_{z}$ is measured) the object is translated only in the direction of the normal to the object surface, i.e., in the direction of $z$-axis.

In general, it is possible to determine all three-translation components $a_{x}, a_{y}, a_{z}$ either simultaneously or subsequently. For example, by means of an arrangement which is similar to the one in Fig. 1, but either with three spatially different observation directions and one illumination direction or with three illumination directions and one observation direction. Obviously it is possible to combine the both mentioned cases, too.

As mentioned above Eqs. (1)-(4) are valid on condition that the rotation and deformation components can be neglected. It is important to look for new solutions in the cases in which this assumption is not fulfilled.

For example, for the determination of the object normal translation $a_{z}$ it is convenient to use the arrangement according to Fig. 2. Two identical linear CCD detectors are placed symmetrically to the $z$-axis and the point source $S$ lies on the $z$-axis. The symmetrical arrangement of observation directions is given by the angles $\theta_{o}$ and $-\theta_{o}$ and by the distance $L_{o}$ from the object point $P$. Providing that the assumptions mentioned at the beginning of this chapter are taken into account and 
the deformation component of the small deformation tensor is omitted only the translation component $a_{z}$ can be determined by means of displacement difference (Eq. (5) for the case of free space and Eq. (6) for the case of image field) of two speckle fields detected in the $\left(X_{1}, Y_{1}\right)$ and $\left(X_{2}, Y_{2}\right)$ planes:

$$
\begin{aligned}
& \Delta A_{X}=A_{X}\left(\theta_{s}, \theta_{o}\right)-A_{X}\left(\theta_{s},-\theta_{o}\right)=-2 a_{z} \sin \theta_{o}, \quad \theta_{s}=0, \\
& \Delta A_{X}=A_{X}\left(\theta_{s}, \theta_{o}\right)-A_{X}\left(\theta_{s},-\theta_{o}\right)=-2\left(\frac{L_{p}^{\prime}-f^{\prime}}{f^{\prime}}\right) a_{z} \sin \theta_{o}, \quad \theta_{s}=0 .
\end{aligned}
$$

The magnitude $\Delta A_{X}$ in Eqs. (5) and (6) corresponds to the difference between the displacements of speckle fields detected in the $\left(X_{1}, Y_{1}\right)$ and $\left(X_{2}, Y_{2}\right)$ planes, quantities
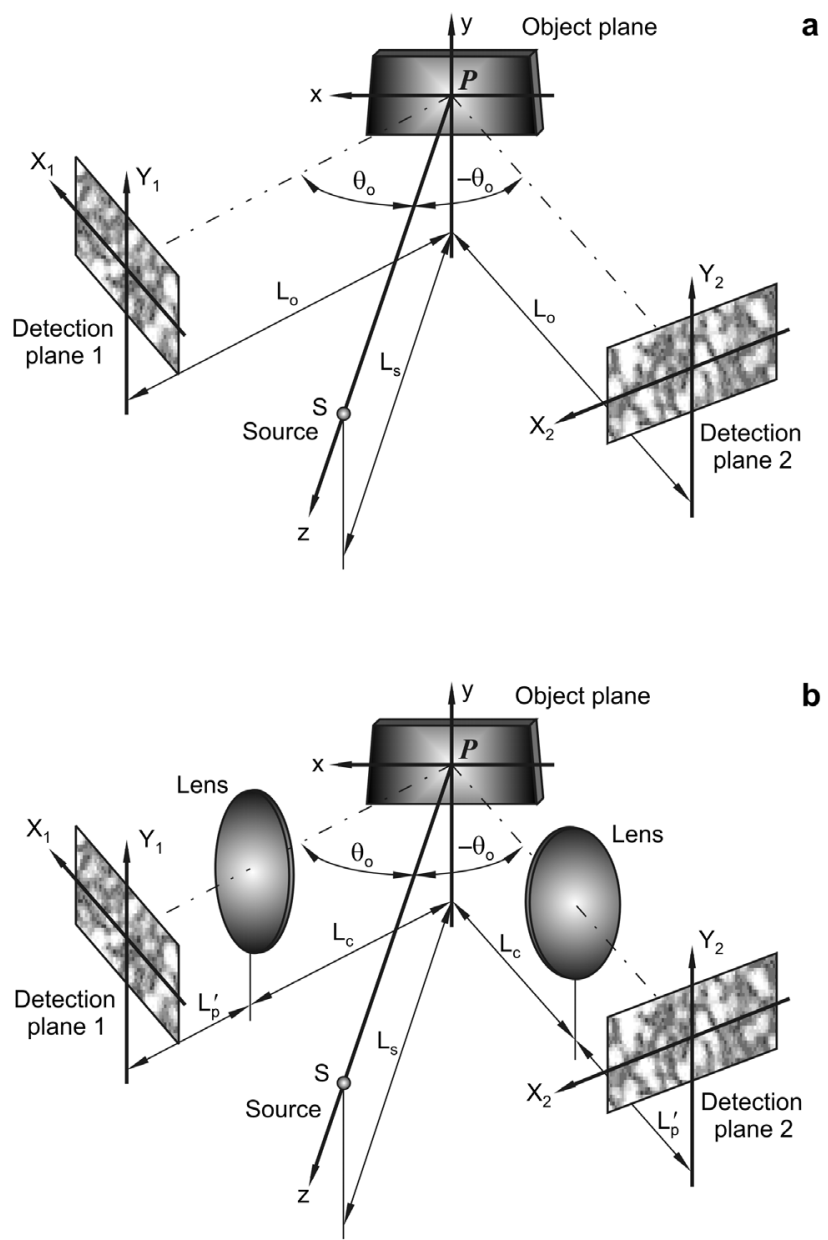

Fig. 2. Geometrical arrangement for the measurement of the object normal translation: a - free space, b - image field. 
$f^{\prime}$ and $L_{p}^{\prime}$ in Eq. (6) represent the focal length of the thin lens and the distance from the thin lens to the detection plane (see Fig. 2b).

In contrast to the previous arrangement (Fig. 1), for this one (Fig. 2) the rotation component of the small deformation tensor need not be neglected. This component eliminates itself during the deriving process of the displacement difference of the two speckle fields [4], [8]-[10].

Another possible solution to determine the normal translation ( $a_{z}$ component) can be based on arrangements that are similar to those in Fig. 2 and that contain two symmetrically placed identical sources and one detector only [9], [10]. If both types of arrangements are compared from the practical point of view the arrangements with two detectors appear to be more suitable for experiments since it is difficult to acquire two identical light sources (as far as beam geometry is concerned).

\section{Theoretical sensitivity and range of measurement analysis}

Now, let us shortly consider the sensitivity and the range of measurement of translation components $a_{x}$ and $a_{z}$ for the arrangement according to Fig. 1. The measurement sensitivity and range depend crucially on the geometrical configuration of the experimental setup (on the geometrical parameters $L_{o}, L_{s}, \theta_{o}, \theta_{s}$ ) and the resolution power of the linear detector used for the measurement (the size of the pixel). The optical system used has also great influence upon results of the measurement. In the case of a thin lens the sensitivity is controlled by the focal length $f^{\prime}$ and the lateral magnification of the thin lens, respectively. This follows from Eq. (3). By virtue of numerical analysis performed for different values of geometrical parameters $L_{s}, L_{o}, L_{c}, L_{p}^{\prime} \in(0.1,1) \mathrm{m}, \theta_{s}, \theta_{o} \in(0,60)^{\circ}$, for the possible range of the detected speckle pattern displacement $A_{X} \in(1,1000)$ pixel (pixel size is $14 \times 14 \mu \mathrm{m}$ ) and various focal lengths of the thin lens $\left(f^{\prime}=19.96 \mathrm{~mm}, f^{\prime}=50 \mathrm{~mm}\right)$ it was found out that theoretical range of measurement of both translation components $a_{x}$ and $a_{z}$ is $10^{-1}-10^{3} \mu \mathrm{m}$. The theoretical analysis also shows that for practical measurements of the translations within the limits from tens to hundreds of $\mu \mathrm{m}$ in the case of free space and within the limits from units to hundreds of $\mu \mathrm{m}$ in the case of image field the theoretically determined relative error is less than $10 \%$. These findings follow from the theory of errors.

Let us analyse the sensitivity and the range of the measurement of translation component $a_{z}$ for the arrangement according to Fig. 2 [10]. If the experimental arrangement for the case of free space with geometrical parameters within the intervals demonstrated in Fig. 3a is considered, i.e., $\theta_{o} \in(10,60)^{\circ}, L_{o} \in(0.2,0.5) \mathrm{m}$ and $\Delta A_{X}=1$ pixel, the sensitivity of the $a_{z}$ quantity measurement lies within the limits $a_{z \min } \in(8.1,40.3) \mu \mathrm{m}$. However, for the case of image field (the thin lens with $\left.f^{\prime}=19.96 \mathrm{~mm}, L_{p}^{\prime} \in(0.2,0.5) \mathrm{m}\right)$ the sensitivity is $a_{z \min } \in(0.3,4.5) \mu \mathrm{m}$ (Fig. $\left.3 \mathbf{b}\right)$. It is evident that the sensitivity of the measurement is strongly increased by using the 


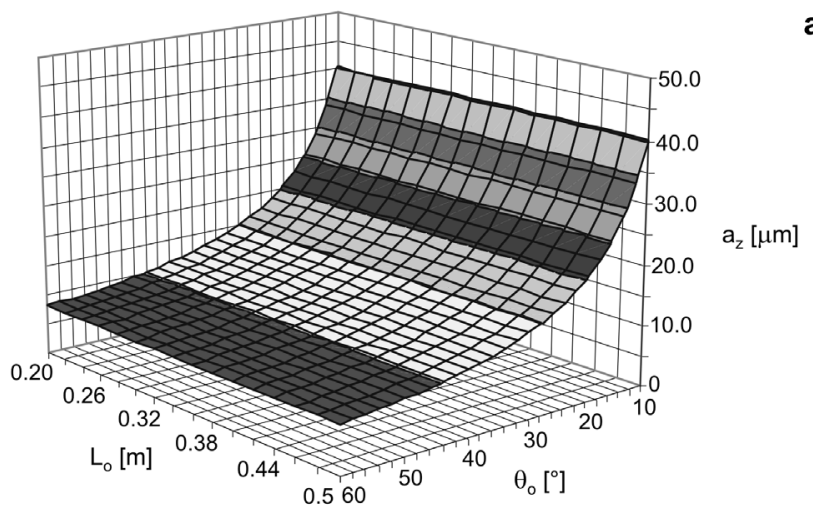

a

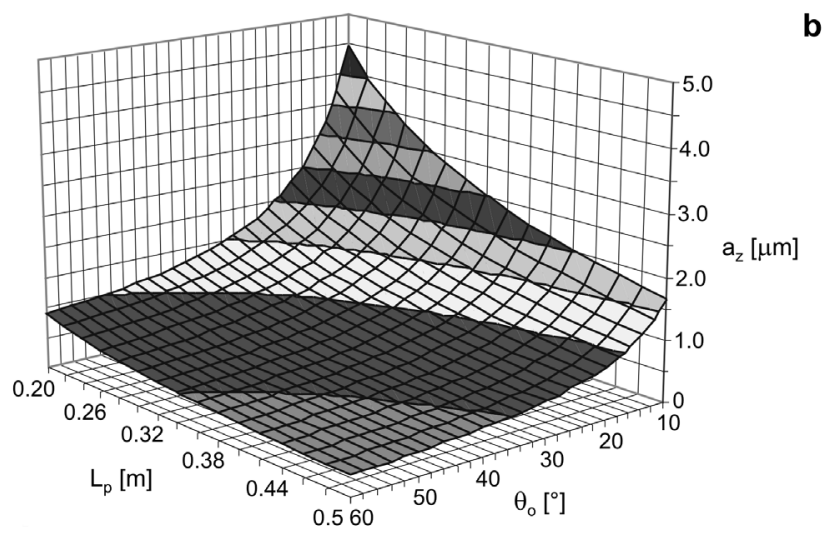

b

Fig. 3. Sensitivity of $a_{z}$ measurement for arrangement shown in Fig. 2: $\mathbf{a}$ - free space, $\mathbf{b}-$ image field $\left(f^{\prime}=19.96 \mathrm{~mm}\right)$.

thin lens. Next, the maximum sensitivity $a_{z \min }=0.3 \mu \mathrm{m}$ can be achieved for the arrangement: $\theta_{o}=60^{\circ}, L_{p}^{\prime}=0.5 \mathrm{~m}$. It is also obvious that the sensitivity grows with the increasing distance $L_{p}^{\prime}$ and the angle $\theta_{o}$. On the contrary, in the case of free space the distance $L_{o}$ from the detectors to the object has theoretically no influence on the sensitivity. On the basis of our sensitivity analysis it is also ascertained that the increasing focal length $f^{\prime}$ decreases the measurement sensitivity $\left(e . g\right.$., $a_{z \min }=0.9 \mu \mathrm{m}$ for $f^{\prime}=50 \mathrm{~mm}$ ). It is theoretically possible to measure the normal component $a_{z}$ within the range $10^{-1}-10^{3} \mu \mathrm{m}$ by means of the arrangement in Fig. 2. This is similar to the results for arrangement in Fig. 1. The theoretical analysis performed also shows that for practical measurements of the translations within the limits from units to hundreds of $\mu \mathrm{m}$ in the case of image field the theoretically determined relative error is less than $15 \%$. 


\section{Experimental setups and results of measurement}

\subsection{Static in-plane translation measurement}

For the measurement of in-plane static object translations ( $x$-axis direction was chosen) the experimental setup, as outlined in Fig. 4, was designed. He-Ne laser was used as a source of coherent light and its beam was directed upright to the investigated object by means of a plane mirror and focused by a lens into a point approximately. The object was represented by cuboid made from different materials (aluminum, steel, pumice, etc.) and placed on a linear stage mounted to a rotary stage. A linear CCD detector (2048 pixels, pixel size $14 \times 14 \mu \mathrm{m}$ ) captured the arising speckle field. The data obtained was then transferred to a PC and processed consecutively.

The measurement process itself proceeded in the following steps. The electronic linear stage with the object was translated several times in the $x$-axis direction by a constant distance with an accuracy of $0.1 \mu \mathrm{m}$. The sample under investigation was returned to the reference state between each translation. The arising speckle field was captured by the CCD detector before and after each translation. So, the finite number of intensity sets was obtained. Then, these sets were numerically processed on a PC by a program that determined the maximum position of the normalized cross-correlation function according to the following relation:

$$
r_{12}(\Delta q)=\frac{\left\langle\Delta I_{1}(q) \Delta I_{2}(q+\Delta q)\right\rangle}{\sqrt{\left\langle\left(\Delta I_{1}(q)\right)^{2}\right\rangle} \sqrt{\left\langle\left(\Delta I_{2}(q)\right)^{2}\right\rangle}}
$$

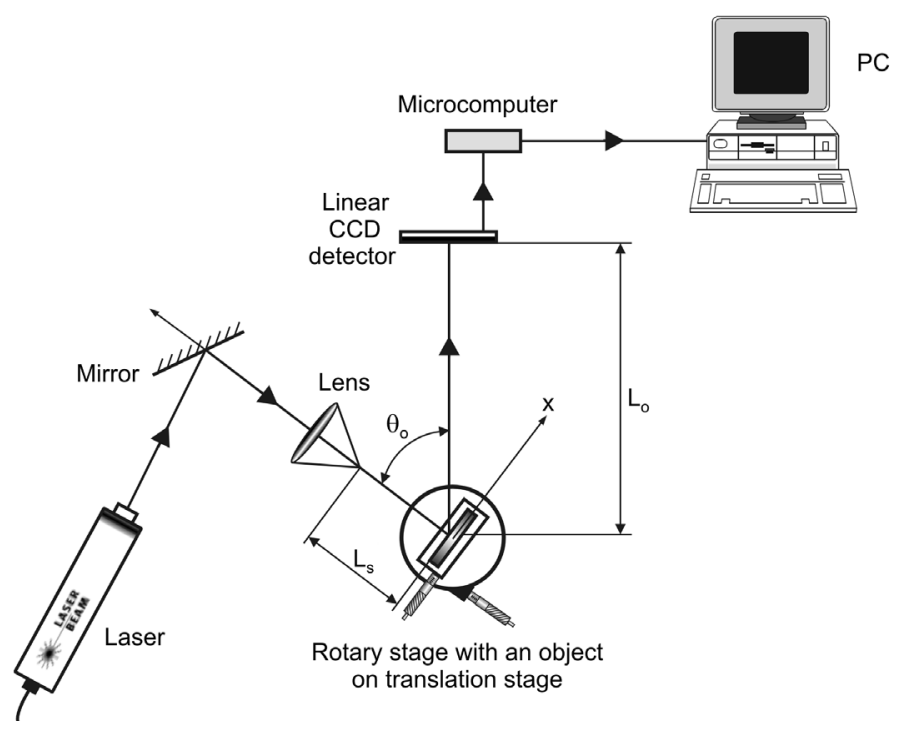

Fig. 4. Experimental setup designed for the noncontact measurement of object in-plane translation in free space. 
$\mathrm{T}$ a b 1 e 1. Translation by $2 \mu \mathrm{m}$ (image field).

\begin{tabular}{lllll}
\hline Geometrical configuration & $n$ & $A_{X}[\mathrm{px}]$ & $r_{1,2}[\%]$ & $a_{x}[\mu \mathrm{m}]$ \\
\hline$L_{p}^{\prime}=441 \mathrm{~mm}$ & 1 & 4 & 96 & 2.3 \\
$L_{c}=110 \mathrm{~mm}$ & 2 & 3.6 & 96.1 & 2.1 \\
$L_{s}=400 \mathrm{~mm}$ & 3 & 3.7 & 96 & 2.1 \\
$\theta_{O}=24^{\circ}$ & 4 & 3.5 & 95.5 & 2.0 \\
$\theta_{s}=0^{\circ}$ & 5 & 3.7 & 95.7 & 2.1 \\
$f^{\prime}=19.96 \mathrm{~mm}$ & 6 & 3.7 & 95.6 & 2.1 \\
& 7 & 3.6 & 95.3 & 2.1 \\
& 8 & 3.8 & 94.8 & 2.2 \\
& 9 & 3.5 & 95.4 & 2.0 \\
& 10 & 3.2 & 95.6 & 1.8 \\
\hline Aluminum object, focusing lens $(f=200 \mathrm{~mm})$. & & $a_{x}=(2.08 \pm 0.04) \mu \mathrm{m}$ \\
\hline
\end{tabular}

$\mathrm{T}$ a b 1 e 2. Results of other measurements of $a_{x n}$ for different geometrical configurations $\left(G_{n}\right.$ while $n=$ $1,2,3)$ and translations $T$.

\begin{tabular}{lll}
\hline$G_{n}$ & $T[\mu \mathrm{m}]$ & $a_{x}[\mu \mathrm{m}]$ \\
\hline$G_{1}$ & 10 (free space) & $-10.0 \pm 0.1$ \\
$G_{2}$ & 30 (image field) & $32.48 \pm 0.04$ \\
$G_{3}$ & 200 (free space) & $-202.8 \pm 0.1$ \\
\hline
\end{tabular}

Legend

$G_{1}$ means: $L_{o}=552 \mathrm{~mm}, L_{s}=540 \mathrm{~mm}, \theta_{o}=24^{\circ}, \theta_{s}=0^{\circ}, f=200 \mathrm{~mm}$;

$G_{2}$ means: $L_{p}^{\prime}=441 \mathrm{~mm}, L_{c}=110 \mathrm{~mm}, L_{s}=400 \mathrm{~mm}, \theta_{o}=24^{\circ}, \theta_{s}=0^{\circ}, f^{\prime}=19.96 \mathrm{~mm}, f=200 \mathrm{~mm}$;

$G_{3}$ means: $L_{o}=552 \mathrm{~mm}, L_{s}=640 \mathrm{~mm}, \theta_{o}=24^{\circ}, \theta_{s}=0^{\circ}, f=200 \mathrm{~mm}$.

where quantities $\Delta I_{1}(q), \Delta I_{2}(q)$ represent sets of fluctuation of intensities of light captured before and after the object deformation and $\Delta q$ is a mutual displacement between the sets $\Delta I_{1}(q), \Delta I_{2}(q)$.

Extensive measurements with objects made from different materials, various geometrical parameters of the arrangement and translation ranges (from units to hundreds of $\mu \mathrm{m}$ ) were done. Let us present some examples of them for clarity. The values of the measured translations are shown in Tab. 1 and in reduced version in Tab. 2. The last version has been obtained by applying the same procedure for different cases (see legend $G_{n}$ under Tab. 2). The translations are $2 \mu \mathrm{m}, 30 \mu \mathrm{m}$ (for the case of image field) and $10 \mu \mathrm{m}, 200 \mu \mathrm{m}$ (for the case of free space).

Let us explain the meaning of every item in the tables. The geometrical configuration involves concrete values of quantities $L_{o}, L_{s}, L_{c}, L_{p}^{\prime}$ in millimeters, $\theta_{o}$, $\theta_{s}$ in degrees and $f^{\prime}$ in millimeters. This is in accordance with Fig. 4 and Fig. 1 b. The measurements were repeated 10 times. $A_{X}$ represents the speckle displacement in pixels (px) determined from the position of the cross-correlation function maximum. In order to find the position of the maximum with the accuracy of $1 / 10$ of the distance 
between two adjacent pixels of the CCD detector the linear interpolation with step $0.1 \mathrm{px}$ was used for approximate evaluation of intensity values between the pixels. Correlation degree $r_{1,2}$ denotes maximal similarity measure, in percents, of two sets of fluctuation of intensities captured before and after the object displacement. The detected translation $a_{x}$ represents the translation in $\mu \mathrm{m}$ evaluated according to Eqs. (1) or (3). Since the sample was translated only in the direction of the $x$-axis the $a_{z}$ component in Eqs. (1) and (3) can be vanished. The last row of the Tab. 1 contains the material of the sample, the focal length of the lens used for focusing of laser beam towards the sample surface and the evaluated average mean value of the detected translation and its standard deviation.

\subsection{Static normal translation measurement}

The experiment as illustrated in Fig. 5 was designed for the practical measurement of the static object normal translation. Next, it will be shown that this optical setup is available for dynamic normal translation measurement, too.

Components used in the experiment were similar as in the previous case: $\mathrm{He}-\mathrm{Ne}$ laser as a light source, aluminum or steel cuboid with a rough surface as an object investigated, pairs of linear CCD cameras $(2048$ pixels, pixel size $14 \times 14 \mu \mathrm{m})$ as detectors, rotary stage and electronic linear stage with an accuracy of translation $0.1 \mu \mathrm{m}$. By means of plane mirrors M1 and M2 the laser beam was directed upright to

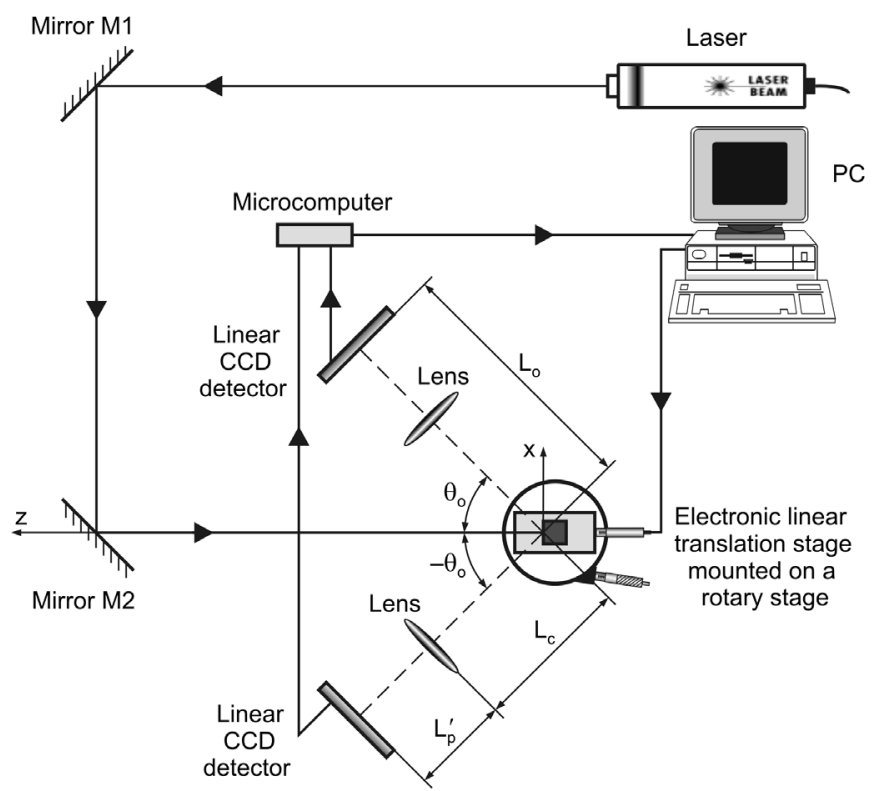

Fig. 5. Experimental setup designed for the noncontact measurement of object normal translation in image field. 
$\mathrm{T}$ a b 1 e 3. Translation by $5 \mu \mathrm{m}$ (image field).

\begin{tabular}{llllllll}
\hline $\begin{array}{lllll}\text { Geometrical } \\
\text { configuration }\end{array}$ & $n$ & \multicolumn{5}{c}{ Detector 1 } & \multicolumn{3}{c}{ Detector 2 } \\
\cline { 2 - 6 }$A_{X 1}[\mathrm{px}]$ & ${ }^{(1)} r_{1,2}[\%]$ & $A_{X 2}[\mathrm{px}]$ & ${ }^{(2)} r_{1,2}[\%]$ & $\Delta A_{X}=A_{X 1}-A_{X 2}[\mathrm{px}]$ & $a_{z}[\mathrm{~mm}]$ \\
\hline$L_{p}^{\prime}=441 \mathrm{~mm}$ & 1 & 2.9 & 97.9 & -3 & 98.7 & 5.9 & 4.8 \\
$\theta_{o}=24^{\circ}$ & 3.2 & 98 & -2.5 & 98.8 & 5.7 & 4.7 \\
$q_{s}=0^{\circ}$ & 3 & 3.2 & 97.7 & -2.7 & 98.6 & 5.9 & 4.8 \\
$f^{\prime}=19.96 \mathrm{~mm}$ & 4 & 2.6 & 98.1 & -3.3 & 98.6 & 5.9 & 4.8 \\
& 5 & 3 & 98.2 & -3 & 98.8 & 6 & 4.9 \\
& 6 & 2.9 & 98 & -2.9 & 98.4 & 5.8 & 4.7 \\
& 7 & 2.9 & 97.9 & -2.9 & 99 & 5.8 & 4.7 \\
& 8 & 2.5 & 98.2 & -3.5 & 98.7 & 6 & 4.9 \\
& 9 & 2.6 & 97.9 & -3.3 & 98.7 & 5.9 & 4.8 \\
\hline
\end{tabular}

$\mathrm{T}$ a b 1 e 4. Results of other measurements of $a_{z}$ for different geometrical configurations $\left(G_{n}\right.$ while $n=$ $1,2,3)$ and translations $T$.

\begin{tabular}{llr}
\hline$G_{n}$ & $T[\mu \mathrm{m}]$ & $a_{z}[\mu \mathrm{m}]$ \\
\hline$G_{1}$ & 50 (free space) & $-49.1 \pm 0.3$ \\
$G_{2}$ & 100 (free space) & $-100.0 \pm 0.4$ \\
$G_{3}$ & 600 (image field) & $599.4 \pm 0.1$ \\
\hline
\end{tabular}

Legend

$G_{1}$ means: $L_{o}=552 \mathrm{~mm}, \theta_{o}=24^{\circ}, \theta_{s}=0^{\circ}$;

$G_{2}$ means: $L_{o}=552 \mathrm{~mm}, \theta_{o}=24^{\circ}, \theta_{s}=0^{\circ}$;

$G_{3}$ means: $L_{p}^{\prime}=442 \mathrm{~mm}, \theta_{o}=24^{\circ}, \theta_{s}=0^{\circ}, f^{\prime}=19.96 \mathrm{~mm}$.

the object $\left(\theta_{s}=0^{\circ}\right)$. The speckle pattern was captured by both cameras simultaneously and the recorded readings were then processed by a program on PC. The PC was able to control the electronic linear stage, too.

Many measurements for various geometrical parameters of arrangements and translation ranges (from units to hundreds of $\mu \mathrm{m}$ ) were done again. Some values of the measured translation are presented in Tab. 3 and, in reduced version, in Tab. 4, which show the translation measurements by $5 \mu \mathrm{m}, 600 \mu \mathrm{m}$ (for the case of image field) and $50 \mu \mathrm{m}, 100 \mu \mathrm{m}$ (for the case of free space). The meaning of every item in the tables is the same as in the above-mentioned section. Since there are two CCD detectors used in the experiment two speckle displacements $A_{X 1}, A_{X 2}$ and their corresponding correlation degrees ${ }^{(1)} r_{1,2},{ }^{(2)} r_{1,2}$ are obtained. The geometrical parameters are in accordance to Fig. 5. The detected translation $a_{z}$ represents the translation in $\mu \mathrm{m}$ evaluated according to Eqs. (5) or (6). 


\subsection{Dynamic translation measurement}

As mentioned above the optical setup in Fig. 5 is convenient for the dynamic translation measurements, too (e.g., velocity and vibration measurements). So far, the case of in-plane velocity has been studied theoretically only [12]. The in-plane velocity of the object investigated can be determined from time-local translation of this object. In the case of vibrations, only the dynamic normal translations were measured in our laboratory. It is, in principle, the determination of temporal behaviour of the normal translation of the object investigated followed by the discrete Fourier analysis. Let us shortly describe the mode of measurement and some of the results obtained.

The experimental setup designed for the noncontact measurement of the normal object vibrations can be seen in Fig. 5. However, the electronic linear stage with the object investigated was substituted by an aluminum disc with a rough surface
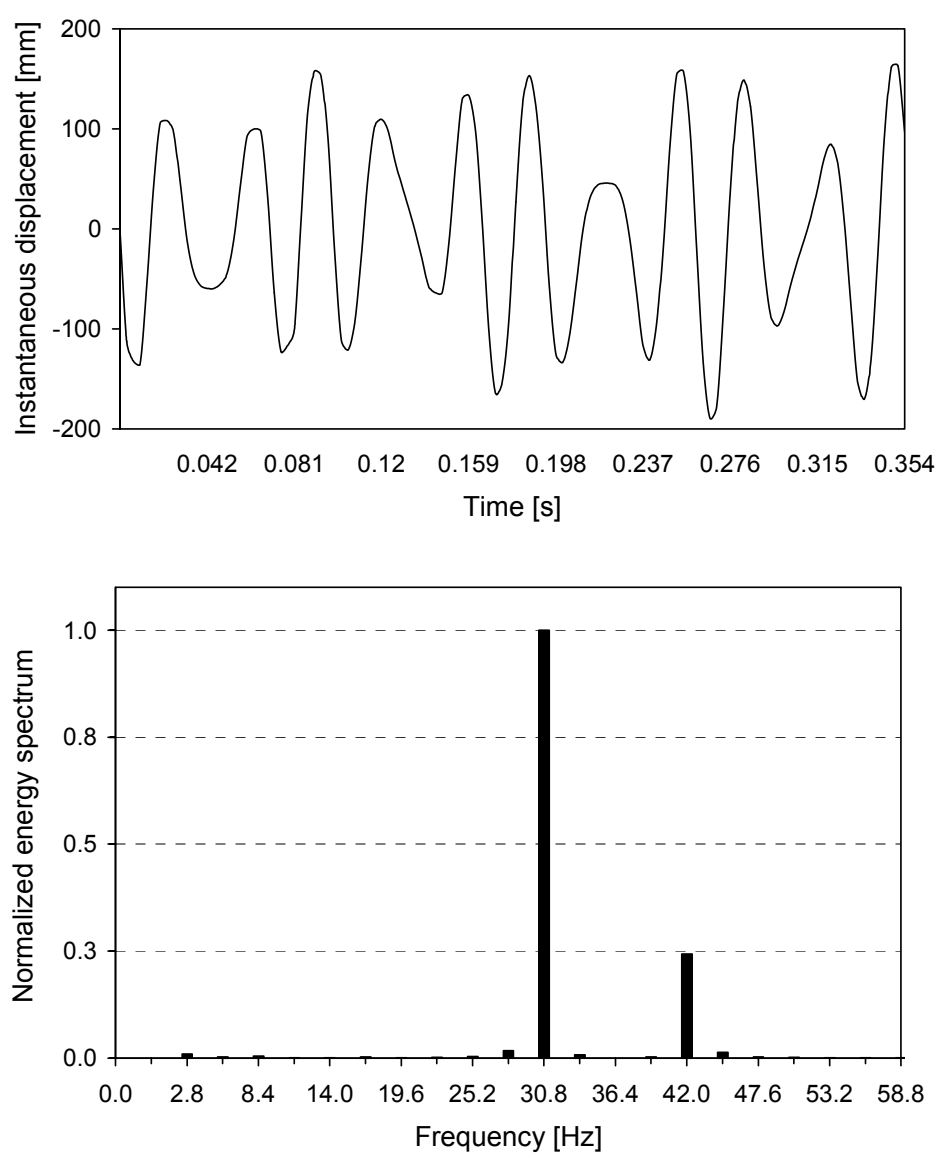

b

Fig. 6. Experimental results; $\mathbf{a}$ - temporal behaviour of instantaneous vibrations displacement of the loudspeaker, b - Fourier analysis. Optical setup: $L_{p}^{\prime}=312 \mathrm{~mm}, \theta_{o}=20^{\circ}$, focal length $f^{\prime}=19.96 \mathrm{~mm}$, loudspeaker driving frequency $31 \mathrm{~Hz}+42 \mathrm{~Hz}$. 


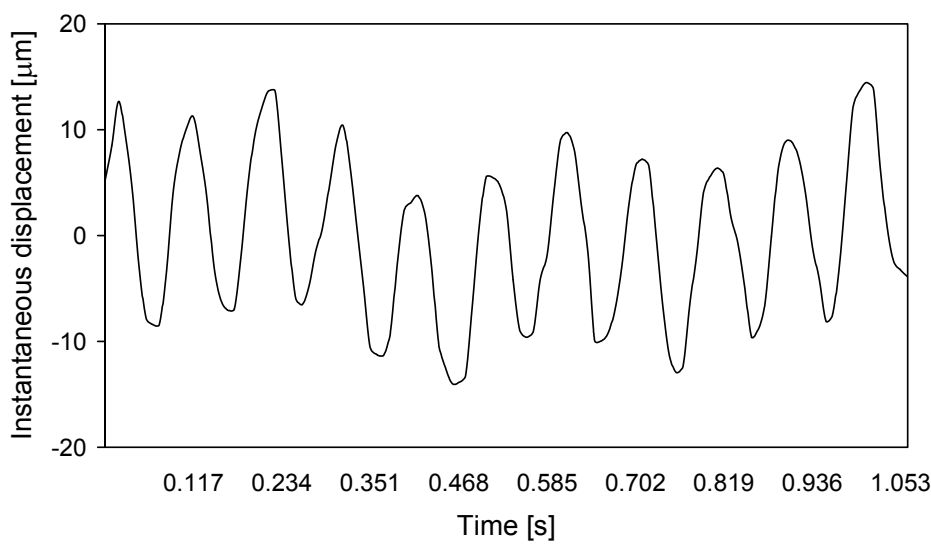

a

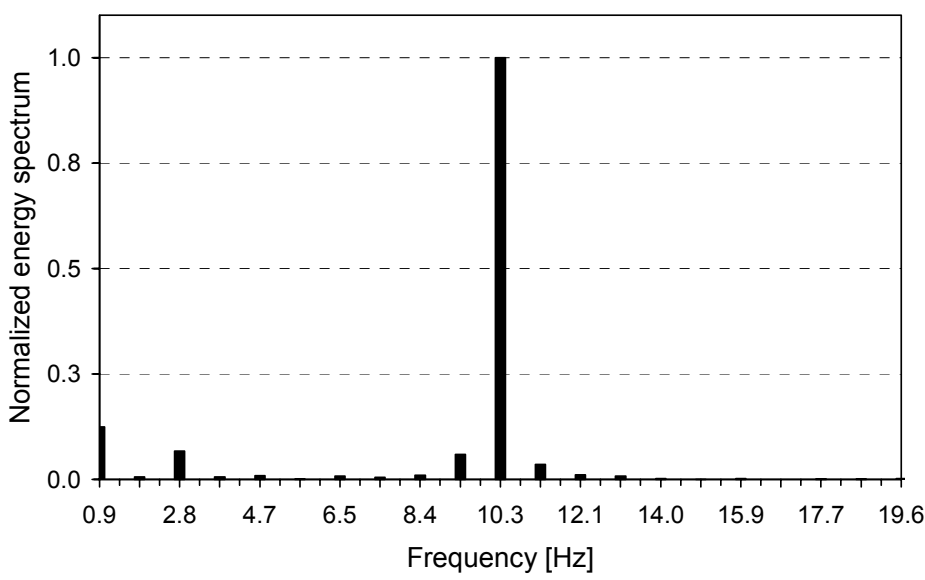

Fig. 7. Experimental results; a - temporal behaviour of instantaneous vibrations displacement of the loudspeaker, b - Fourier analysis. Optical setup: $L_{p}^{\prime}=441 \mathrm{~mm}, \theta_{o}=24^{\circ}$, focal length $f^{\prime}=19.96 \mathrm{~mm}$, loudspeaker driving frequency $10 \mathrm{~Hz}$.

mounted on a loudspeaker. The loudspeaker vibrated with a frequency and amplitude controlled by a PC soundcard. The amplitude magnitude was checked by a record player pick-up, too.

Detection of the vibrating frequency was our primary goal. Therefore, extensive experimental measurements were carried out with different loudspeaker driving frequencies. The object was driven by a sine signal with a simple frequency, a signal represented by a superposition of two sine signals and three sine signals and by a sine signal with a simple frequency, onto which the Gaussian noise was modulated. We present only some of them in this paper, that is, superposition of two sine signals with frequencies $31 \mathrm{~Hz}$ and $42 \mathrm{~Hz}$ (Fig. 6), sine signal with simple frequency $10 \mathrm{~Hz}$ (Fig. 7) and sine signal with simple frequency $5 \mathrm{~Hz}$ (Fig. 8). The temporal behaviour of normal translation of the object investigated obtained during the data processing gave us additional information on the vibration amplitude. The results of the static measure- 


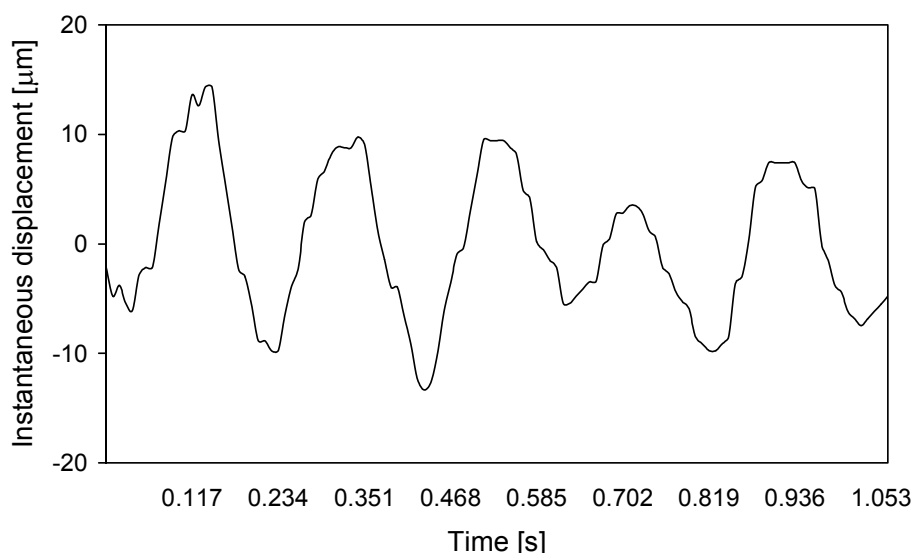

a

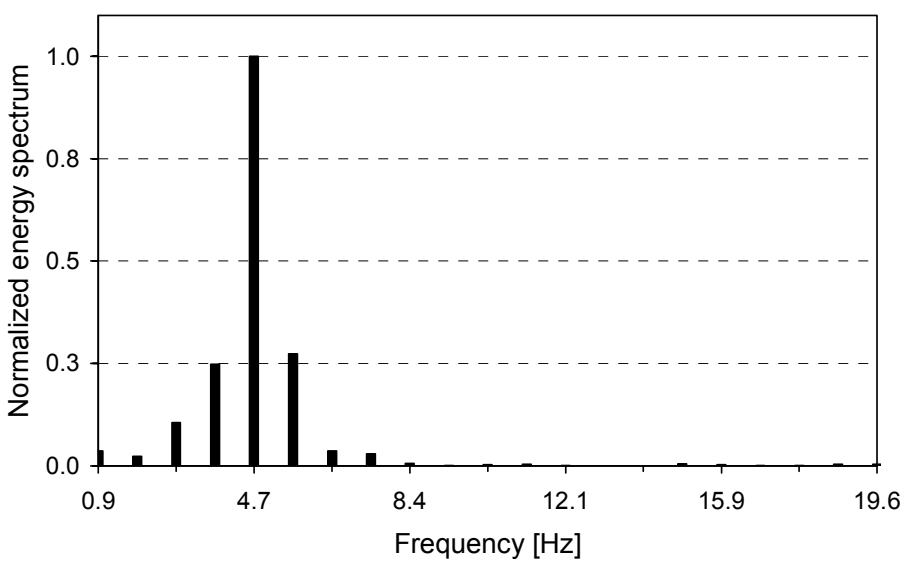

b

Fig. 8. Experimental results; a - temporal behaviour of instantaneous vibrations displacement of the loudspeaker, b - Fourier analysis. Optical setup: $L_{p}^{\prime}=441 \mathrm{~mm}, \theta_{o}=24^{\circ}$, focal length $f^{\prime}=19.96 \mathrm{~mm}$, loudspeaker driving frequency $5 \mathrm{~Hz}$.

ments presented in the previous section confirm the possibility of determining correct amplitude.

Our results for the various vibrating frequencies are plotted in several pairs of graphs that represent the evaluated temporal behaviour of the instantaneous displacement of the loudspeaker vibrations and its normalized energy spectrum.

Only results of measurements of vibrations with frequencies higher than $15 \mathrm{~Hz}$ have been published so far [13]. These results are sufficient for multiple applications in industry (e.g., detection of machine vibrations). However, there are other applications that require measurements of vibrations with smaller frequencies, e.g., in area of medicine. One of the applications can be a study of vibrations and movements of human tissue. 
The CCD camera used had limited amount of memory (only for 125 frames) and limited sampling frequency (the lowest sample frequency was $111 \mathrm{~Hz}$ ). Due to this hardware constraint and the fact that the discrete Fourier transform was used for the evaluation of Fourier analysis it was not able to reliably sample vibrations with frequencies smaller than $7 \mathrm{~Hz}$. The high sampling frequency and the small number of frames caused that the frequency step in the Fourier spectra was too large with respect to the measured frequency, thereby very large error occurred in the determination of the object vibration frequencies much smaller than $7 \mathrm{~Hz}$. In order to manifest the consequence of the hardware constraint of CCD cameras used we present results of the measurement of the object vibrations with frequency $5 \mathrm{~Hz}$ (Fig. 8).

\section{Conclusions}

The developing world sets high demands to measuring methods, especially to their non-destructive way of the measurement. It is shown that optics satisfies the requirement. This paper presented a purely optical method that takes advantage of an optical effect considered as a parasitic one for a long time. It is evident that the speckle pattern resulting from the non-destructive interaction of coherent light with environment causing random phase distribution can be used as a mean for determining the state of the environment studied or eventually an object.

The static and dynamic translation measurements have been analyzed in this paper. Different optical setups, measurement ranges and experimental results are presented. The analysis of results confirmed applicability of this method for usage in the translation measurements (both in-plane and normal) within the range from tens to hundreds of $\mu \mathrm{m}$ for the free space case and from units to hundreds of $\mu \mathrm{m}$ for the image field case. The geometrical parameters of the optical setup and the optical system used enable a full control of the measurement sensitivity and range. The experiments of dynamic vibration measurements showed the possibility to measure vibrations both harmonic and inharmonic.

One should note the advantage of this measuring method lying in simple practical realization, using computer technique and modern optoelectronic elements. Therefore, it can be useful for multiple applications both in industry and medicine.

Acknowledgments -This work was supported by the project LN00A015 of the Ministry of Education of the Czech Republic.

\section{References}

[1] DAINTy J.C., Laser Speckle and Related Phenomena, Springer-Verlag, Berlin 1984.

[2] Frieden B., Probability, Statistical Optics and Data Testing, Springer-Verlag, New York 1983.

[3] Françon M., Laser Speckle and Application in Optics, Academic Press, New York 1979.

[4] Yamaguchi I., Opt. Acta 28 (1981), 1359. 
[5] Yamaguchi I., J. Phys. E: Sci Instrum. 19 (1986), 944.

[6] Yamaguchi I., Takemori T., Kobayashi I., Opt. Eng. 32 (1993), 618.

[7] Yamaguchi I., Fujita T., Appl. Opt. 28 (1989), 4401.

[8] Hrabovský M., BaČa Z., Horváth P., Opt. Lasers Eng. 32 (2000), 395.

[9] Hrabovský M., BaČa Z., Horváth P., Czech. J. Phys. 51 (2001), 129.

[10] Horváth P., HrabovskÝ M., Šmíd P., J. Modern Opt. 51 (2004), 725.

[11] Hrabovsky M., Baca Z., Horvath P., Optik 111 (2000), 359.

[12] Hrabovský M., Horváth P., BaČa Z., Šmíd P., Soukup L., Proc. SPIE 4448 (2001), 380.

[13] Hrabovský M., Šmíd P., Horváth P., BaČa Z., Optik 113 (2002), 117.

[14] Jones R., Wykes C., Holographic and Speckle Interferometry, 2nd ed., Cambridge University Press, Bristol 1989. 